\title{
Difference in the Contents of Music Intervention to Control Agitation by Music Providers
}

\author{
Heeok Park \\ College of Nursing, Keimyung University, Daegu, South Korea \\ Email: hopark@kmu.ac.kr
}

Received 16 July 2015; accepted 25 August 2015; published 28 August 2015

Copyright (C) 2015 by author and Scientific Research Publishing Inc.

This work is licensed under the Creative Commons Attribution International License (CC BY).

http://creativecommons.org/licenses/by/4.0/

c) (i) Open Access

\section{Abstract}

Purpose: The purpose of this study was to compare the contents of music intervention studies between the music therapists and non-music therapists who endeavored to control agitation in patients with dementia, and to provide meaningful ideas to improve the music interventions by music providers. Method: This study is a review study by searching CINAHL, MEDLINE, and PsychINFO for the keywords, "agitation" and "music," which are used in the searches. Results: A total of 30 studies of music intervention ( 7 studies about music therapists and 23 studies about nonmusic therapists) were included for the review. The studies about music therapists had a more reasonable sample size, variety of music activities, and comparison groups. The studies of nonmusic therapists were provided by nurses, researchers, recreational therapists, and trained nursing assistants and they provided music using background music at a scheduled time for care, such as mealtimes and bathing times. Conclusions: Studies on music interventions need to have a more rigorous research design, such as randomized controlled trials for the future studies. Furthermore, there is a need for multi-disciplinary music intervention studies by music providers who have different types of educational backgrounds and clinical experiences.

\section{Keywords}

Music, Agitation, Dementia

\section{Introduction}

Persons with dementia have health problems that include cognitive decline, language deterioration, poor judgment, restlessness, confusion, and changes in social-behavioral problems [1]. Among these problems, agitation 
has become a major issue because it increases nursing-home placement, health maintenance costs, and the burdens of caregivers [2]-[4]. Pharmacological interventions are often necessary to control agitation in persons with dementia, but they bring about undesirable effects such as a high risk of fall, declined cognition levels, and monetary burdens [5] [6]. To reduce the undesirable effects of pharmacological interventions, music intervention is currently being provided to control agitation because it is relatively convenient, non-pervasive, and costeffective. Music therapy is defined as "an established allied health profession using music and music activities to address physical, psychological, cognitive and social needs of individuals with disabilities” [7].

Many studies have reported the positive effects of music intervention on diverse health problems in persons with dementia, including cognitive decline, less-alert responses, lack of engagement between caregiver and carereceiver, disruptive behaviors, impaired language function, inattention, lack of food intake, and poor social relationships [8]-[13]. Many studies have also reported the positive effects of music intervention on agitation in persons with dementia [14]-[18]. Music intervention consists of music group activities, listening to music, singing, or playing instruments [9] [16] [17]. Most music-intervention studies have been performed in nursing facilities, with only one study that was conducted at the client's residence [17].

Music intervention to control agitation in persons with dementia has been performed by diverse healthcare providers. The providers can be categorized into two groups: music therapists and non-music therapists (the latter includes nurses, certified nursing assistants, recreation specialists, and family members; [15] [19] [20]. Music therapists are inclined to use different methods of music intervention compared with non-music therapists, reflecting their different educational backgrounds and clinical experiences. Even though music intervention providers tend to use different types of music intervention to control agitation, there has been no description regarding how the interventions are different depending on the different educational backgrounds of the music intervention providers. A few studies reviewed the effects of music intervention on agitation, and a review focused on the findings of music interventions, but not on music contents and music providers [21]-[23].

The present study was undertaken to fill the knowledge gap by examining how the content of music intervention is different between music therapists and non-music therapists based on a review of published research articles. The findings reveal more of the strengths and weaknesses of music intervention by each group, and also how music interventions might be improved. Specifically, the present review focused on how the contents of music intervention used to control agitation in persons with dementia differed between music therapists and nonmusic therapists. This new knowledge should aid in developing more practical guidelines of music intervention for each group of music providers.

\section{Method}

\subsection{Design}

This study is a review study to investigate the contents of music intervention to control agitation in patients with dementia and to compare the contents of music intervention by the intervention providers.

\subsection{Sample Selection}

A review of the research literature was undertaken by searching computerized databases (CINAHL, MEDLINE, and PsychINFO) from 1990-2015 June. Some of the keywords used in the literature search included music and agitated. By the first search, a total of 119 articles were listed. The criteria for article selection were those articles published in English, reported an intervention study using music, and studied controlling agitation in persons with dementia or Alzheimer's disease. Based on the findings of the inclusion criteria, a total of 30 articles were selected. The others were excluded because they were no intervention studies, not performed for patients with dementia, not mainly performed using music intervention, or not mainly provided to control agitation. Thus, the remaining 30 articles were included for the review.

\section{Results}

\subsection{Subjects' Characteristics}

A total of 30 research-based studies were included for the review and the studies were divided into two groups according to the purpose of the proposed study of music therapists and non-music therapists. Among the 30 stu- 
dies, 7 studies were performed by music therapists and the other 23 studies were provided by non-music therapists, including nurses, certified nurse assistants, recreational specialists, and family caregivers. The overall findings of the music-intervention studies were reviewed, including research design, sample size, setting, music interventions, instruments, and outcomes. The overall findings about the music therapists are shown in Table 1 and concerning the non-music therapists in Table 2. The contents of music interventions were reviewed and they included conceptual framework or theoretical framework, music-intervention providers and agitation raters, music-intervention tools, and music-intervention time schedules (timing, duration, frequency, and total sessions). The contents of music interventions by music therapists are presented in Table 3 and non-music therapists in Table 4.

\subsection{The Characteristics of Music Interventions Studies}

\subsubsection{Sample Size and Setting}

In the 30 music-intervention studies to control agitation in persons with dementia that were identified, the sample sizes were diverse and varied from 1 - 100. In the music therapists' studies, the sample size varied from 11 77, with $86 \%$ of the studies having a sample size exceeding 15 (Table 1). In the non-music therapists' studies,

Table 1. Music therapists: the characteristics of music intervention studies to control agitation $(N=7)$.

\begin{tabular}{|c|c|c|c|c|c|c|c|c|c|}
\hline Year & Authors & Design & $\begin{array}{c}\mathrm{SS}^{*} \\
(\mathrm{M} / \mathrm{F})\end{array}$ & $\begin{array}{l}\text { Age range } \\
\text { mean (SD) }\end{array}$ & Setting & $\begin{array}{c}\text { Music } \\
\text { intervention }\end{array}$ & $\begin{array}{l}\text { Dependent } \\
\text { variables }\end{array}$ & Instrument & Outcome \\
\hline 1994 & $\begin{array}{l}\text { Clair \& } \\
\text { Bernstein. }\end{array}$ & $\mathrm{QE}^{*}(\mathrm{ABC})$ & $28(27 / 1)$ & $56-81$ & $\begin{array}{l}\text { A day room, } \\
\text { Veterans Affairs } \\
\text { Medical Center }\end{array}$ & $\begin{array}{l}\text { Group music } \\
\text { activities } \\
\text { (stimulative } \\
\text { music and } \\
\text { sedative music) }\end{array}$ & Agitation & & $\begin{array}{l}\text { No significant } \\
\text { difference } \\
\text { between no music, } \\
\text { stimulative music } \\
\text { and sedative music }\end{array}$ \\
\hline 1996 & $\begin{array}{l}\text { Brotons \& } \\
\text { Pickett-Cooper }\end{array}$ & $\mathrm{QE}$ & $20(3 / 17)$ & $\begin{array}{l}70-96 \\
82(6.57)\end{array}$ & $\begin{array}{l}4 \text { Nursing } \\
\text { homes }\end{array}$ & $\begin{array}{l}\text { Group music } \\
\text { activities }\end{array}$ & $\begin{array}{l}\text { Agitation, } \\
\text { dosages } \\
\text { of PRN } \\
\text { medication }\end{array}$ & $\begin{array}{l}\text { DBRS* } \\
\text { Video } \\
\text { analysis }\end{array}$ & $\begin{array}{l}\text { Significantly more } \\
\text { agitated before music } \\
\text { therapy than during, } \\
\text { and after music } \\
\text { herapy sessions }\end{array}$ \\
\hline 2002 & $\begin{array}{l}\text { Jennings } \\
\text { \&Vance }\end{array}$ & $\mathrm{QE}$ & $16(2 / 14)$ & $\begin{array}{l}78.80 \\
(3.91)\end{array}$ & $\begin{array}{l}\text { An activity } \\
\text { room, } \\
\text { Alzheimer's } \\
\text { adult day care }\end{array}$ & $\begin{array}{l}\text { Group music } \\
\text { therapy: sing } \\
\text { familiar songs, } \\
\text { sing-alongs, } \\
\text { and playing } \\
\text { instrument }\end{array}$ & Agitation & M-CMAI* & $\begin{array}{l}\text { Agitation was } \\
\text { significantly reduced } \\
\text { during music therapy } \\
\text { compared to baseline: }\end{array}$ \\
\hline 2003 & $\begin{array}{l}\text { Brotons \& } \\
\text { Marti }\end{array}$ & $\begin{array}{l}\text { One group } \\
\text { pre-test and } \\
\text { post-test }\end{array}$ & $\begin{array}{l}11 \mathrm{pts} \\
\& 11 \\
\text { care-givers }\end{array}$ & & A rural area & $\begin{array}{l}\text { Singing, playing } \\
\text { instrument, } \\
\text { \& movement/ } \\
\text { dance }\end{array}$ & $\begin{array}{l}\text {-Agitation. } \\
\text {-Caregiver's } \\
\text { burden and } \\
\text { depression. }\end{array}$ & $\begin{array}{l}\text { CMAQ,* } \\
\text { Caregivers } \\
\text { burden } \\
\text { questionnaire, } \\
\text { Back's } \\
\text { Depression } \\
\text { Scale }\end{array}$ & $\begin{array}{l}\text { Significant difference } \\
\text { between pre and } \\
\text { post-test scores in } \\
\text { agitation, no significant } \\
\text { difference in the } \\
\text { caregivers' burden and } \\
\text { depression over time }\end{array}$ \\
\hline 2007 & $\begin{array}{l}\text { Ledger \& } \\
\text { Baker }\end{array}$ & $\begin{array}{l}\text { A longitudinal } \\
\text { epeated } \\
\text { measures } \\
\text { design }\end{array}$ & 45 & $71-96$ & $\begin{array}{l}13 \text { Nursing } \\
\text { homes }\end{array}$ & $\begin{array}{l}\text { EG: group } \\
\text { music therapy } \\
\text { CG: no } \\
\text { intervention }\end{array}$ & Agitation & $\begin{array}{l}\text { CMAI* } \\
\text { Interview }\end{array}$ & $\begin{array}{l}\text { No significant } \\
\text { difference between } \\
\text { EG and CG in } \\
\text { agitation over time. }\end{array}$ \\
\hline 2013 & Ridder et al. & $\begin{array}{l}\text { A pragmatic, } \\
\text { two-armed, } \\
\text { cross-over, } \\
\text { exploratory, } \\
\text { randomized } \\
\text { controlled } \\
\text { study }\end{array}$ & 42 & $\begin{array}{l}66-96 \\
(81)\end{array}$ & $\begin{array}{l}14 \text { Nursing } \\
\text { homes }\end{array}$ & $\begin{array}{l}\text { EG: individualized } \\
\text { music therapy } \\
\text { Improvising, } \\
\text { singing, dancing/ } \\
\text { moving, listening, } \\
\text { and other activities) } \\
\text { CG: standard care }\end{array}$ & $\begin{array}{l}\text { Agitation } \\
\text { Medication } \\
\text { QOL }\end{array}$ & $\begin{array}{l}\text { CMAI* } \\
\text { ADROL }\end{array}$ & $\begin{array}{l}\text { Agitation and } \\
\text { prescription of } \\
\text { medication increased } \\
\text { during standard care } \\
\text { and decreased during } \\
\text { music therapy } \\
\text { significantly. }\end{array}$ \\
\hline 2013 & Vink et al. & $\begin{array}{l}\text { A randomized } \\
\text { controlled } \\
\text { design }\end{array}$ & 77 & $\begin{array}{l}82.16 \\
(6.87)\end{array}$ & $\begin{array}{l}\text { Dutch nursing } \\
\text { homes, } \\
\text { Netherlands }\end{array}$ & $\begin{array}{l}\text { EG: music therapy } \\
\text { (singing, dancing, } \\
\text { playing a musici } \\
\text { nstrument etc.) } \\
\text { CG: recreational } \\
\text { activities }\end{array}$ & Agitation & CMAI & $\begin{array}{l}\text { A decrease in agitated } \\
\text { behaviors in both } \\
\text { groups but no } \\
\text { difference between } \\
\text { groups }\end{array}$ \\
\hline
\end{tabular}

*CMAI = Cohen-Mansfield Agitation Inventory; CMAQ = Cohen Mansfield Agitation Questionnaire; DBRS = Disruptive Behavior Rating Scales; M-CMAI = Modified Cohen-Mansfield Agitation Inventory; NPI = Neuropsychiatry Inventory; PGC-IADL = Philadelphia Geriatric Centre Instrumental Activities of Daily Living; SS = sample size. 
Table 2. Non-music therapists: the characteristics of music intervention studies to control agitation $(N=23)$.

\begin{tabular}{|c|c|c|c|c|c|c|c|c|c|}
\hline Year & Authors & Design & $\begin{array}{l}\mathrm{SS}^{*} \\
(\mathrm{M} / \mathrm{F})\end{array}$ & $\begin{array}{l}\text { Age Range } \\
\text { Mean (SD) }\end{array}$ & Setting & $\begin{array}{c}\text { Music } \\
\text { Intervention }\end{array}$ & $\begin{array}{l}\text { Dependent } \\
\text { Variables }\end{array}$ & Instrument & Outcome \\
\hline 1993 & $\begin{array}{l}\text { Gerdner \& } \\
\text { Swanson }\end{array}$ & $\begin{array}{l}\text { A case } \\
\text { study }\end{array}$ & 5 & $70-99$ & $\begin{array}{l}\text { A health } \\
\text { center }\end{array}$ & Individualized music & $\begin{array}{l}\text { Agitation, } \\
\text { \# of medication }\end{array}$ & M-CMAI ${ }^{*}$ & $\begin{array}{l}\text { Agitation decreased during music } \\
\text { intervention in } 4 \text { out of } 5 \text { clients. }\end{array}$ \\
\hline 1994 & $\begin{array}{l}\text { Goddaer \& } \\
\text { Abraham }\end{array}$ & $\mathrm{ABAB}$ & $\begin{array}{c}29 \\
(6 / 23)\end{array}$ & $\begin{array}{c}69-93 \\
81.3(6.9)\end{array}$ & $\begin{array}{l}2 \text { nursing } \\
\text { homes, }\end{array}$ & $\begin{array}{l}\text { A: no music } \\
\text { B: Group music } \\
\text { (Relaxing music) }\end{array}$ & Agitation & M-CMAI. & $\begin{array}{l}\text { Agitation significantly decreased } \\
54 \% \text { with music compared to } \\
\text { baseline and increased } 38.4 \% \\
\text { when withdrawal period }\end{array}$ \\
\hline 1995 & $\begin{array}{l}\text { Tabloski } \\
\text { et al. }\end{array}$ & $\mathrm{QE}^{*}$ & $\begin{array}{c}20 \\
(3 / 17)\end{array}$ & $64-84$ & $\begin{array}{l}2 \text { Long term } \\
\text { care facilities }\end{array}$ & Group (Calming music) & Agitation & $\begin{array}{l}\text { Agitation } \\
\text { Behavior } \\
\text { Scale }\end{array}$ & $\begin{array}{l}\text { A significant reduction in agitated } \\
\text { both during and after music } \\
\text { intervention compared to baseline }\end{array}$ \\
\hline 1996 & $\begin{array}{l}\text { Ragneskog } \\
\text { et al. }\end{array}$ & & 5 & $69-85$ & $\begin{array}{l}\text { A nursing } \\
\text { home }\end{array}$ & $\begin{array}{l}\text { Group (Soft, Swedish, } \\
\text { Pop/rock music }\end{array}$ & Agitation & $\begin{array}{l}\text { Video } \\
\text { analysis. }\end{array}$ & $\begin{array}{l}\text { Two of the five patients didn't } \\
\text { show restless during soothing } \\
\text { music condition. }\end{array}$ \\
\hline 1997 & Denney & $\mathrm{QE}$ & $\begin{array}{c}9 \\
(3 / 6)\end{array}$ & $\begin{array}{c}65-84 \\
74.8(6.4)\end{array}$ & $\begin{array}{l}\text { A nursing } \\
\text { home }\end{array}$ & Quiet music & Agitation. & M-CMAI & $\begin{array}{l}\text { Agitation decreased with } \\
\text { music from baseline }\end{array}$ \\
\hline 1997 & Gerdner & $\begin{array}{l}\text { A case } \\
\text { study }\end{array}$ & 1 & 77 & & $\begin{array}{l}\text { Classical-relaxation } \\
\text { \& Preferred music } \\
\text { (spiritual or } \\
\text { religious music) }\end{array}$ & Agitation & M-CMAI & $\begin{array}{l}\text { Agitation decreased during } \\
\text { preferred music condition } \\
\text { than classical music }\end{array}$ \\
\hline 1997 & $\begin{array}{l}\text { Thomas, } \\
\text { Heitman, \& } \\
\text { Alexander. }\end{array}$ & $\mathrm{QE}$ & $\begin{array}{c}14 \\
(4 / 10)\end{array}$ & $69-86$ & $\begin{array}{l}\text { A nursing } \\
\text { facility }\end{array}$ & $\begin{array}{l}\text { Individualized } \\
\text { and Preferred } \\
\text { music }\end{array}$ & $\begin{array}{l}\text { Aggressive } \\
\text { behaviors }\end{array}$ & M-CMAI & $\begin{array}{l}\text { There were significant differences } \\
\text { on physically aggressive behaviors } \\
\text { between pre-music and post-music } \\
\text { and music and post-music }\end{array}$ \\
\hline 1998 & $\begin{array}{l}\text { Clark, Lipe, } \\
\text { \& Bilbrey }\end{array}$ & QE & $\begin{array}{c}18 \\
(4 / 14)\end{array}$ & $\begin{array}{l}55-95 \\
82(10)\end{array}$ & $\begin{array}{l}\text { A nursing } \\
\text { facility }\end{array}$ & Preferred music & $\begin{array}{l}\text { Aggressive } \\
\text { behaviors. }\end{array}$ & Observation & $\begin{array}{l}\text { The total number of aggressive } \\
\text { ehaviors significantly decreased } \\
\text { with music compared to no music }\end{array}$ \\
\hline 2000 & Gerdner & $\begin{array}{l}\text { A pre and } \\
\text { posttest } \\
\text { crossover } \\
\text { design }\end{array}$ & $\begin{array}{c}39 \\
(9 / 30)\end{array}$ & 82.6 & $\begin{array}{l}6 \text { long-term } \\
\text { care facilities }\end{array}$ & $\begin{array}{l}\text { Individualized M } \\
\text { Vs. Classical-Relaxation } \\
\text { music }\end{array}$ & Agitation & M-CMAI & $\begin{array}{l}\text { Significant reduction in agitation } \\
\text { with individualized music and } \\
\text { even after withdrawal compared } \\
\text { to classical music }\end{array}$ \\
\hline 2004 & $\begin{array}{l}\text { Richeson } \\
\text { \& Neill }\end{array}$ & $\mathrm{QE}$ & $\begin{array}{c}27 \\
(6 / 21)\end{array}$ & $\begin{array}{c}87 \\
(67-94)\end{array}$ & $\begin{array}{l}\text { Nursing } \\
\text { facility }\end{array}$ & Quiet music & $\begin{array}{l}\text { Agitation, } \\
\text { Food intake }\end{array}$ & $\begin{array}{l}\text { M-CMAI, } \\
\text { Percentage } \\
\text { of food eaten }\end{array}$ & $\begin{array}{l}\text { Overall agitation decreased } \\
\text { compared to baseline to } \\
\text { intervention and food eaten } \\
\text { increased from baseline }\end{array}$ \\
\hline 2005 & Gerdner & QE & $\begin{array}{c}8 \\
(0 / 8)\end{array}$ & 83.3 & $\begin{array}{l}\text { A skilled } \\
\text { care facility }\end{array}$ & $\begin{array}{l}\text { Preferred music } \\
\text { individualized }\end{array}$ & Agitation & $\begin{array}{l}\text { M-CMAI } \\
\text { VAS }^{*} \\
\text { Interviews }\end{array}$ & $\begin{array}{l}\text { A significant reduction in agitation } \\
\text { during the presentation of music } \\
\text { from baseline and daytime }\end{array}$ \\
\hline 2005 & $\begin{array}{l}\text { Hicks- } \\
\text { Moore }\end{array}$ & QE & $\begin{array}{c}30 \\
(9 / 21)\end{array}$ & $\begin{array}{c}82.4 \\
(70-101)\end{array}$ & $\begin{array}{l}\text { A nursing } \\
\text { home }\end{array}$ & Relaxing music & Agitation & M-CMAI & $\begin{array}{l}\text { Mean of agitated behaviors } \\
\text { decreased with music }\end{array}$ \\
\hline 2006 & $\begin{array}{l}\text { Sung } \\
\text { et al. }\end{array}$ & $\mathrm{QE}$ & 57 & - & $\begin{array}{l}\text { A residential } \\
\text { care facility }\end{array}$ & $\begin{array}{l}\mathrm{EG}^{*}: \text { Preferred music } \\
\mathrm{CG}^{*}: \text { No music }\end{array}$ & Agitation & $\mathrm{CMAI}^{*}$ & $\begin{array}{l}\text { A significant reduction in overall } \\
\text { CMAI. Physically non-aggressive } \\
\text { behaviors in music group decreased } \\
\text { compared to the no music group }\end{array}$ \\
\hline 2006 & $\begin{array}{l}\text { Sung } \\
\text { et al. }\end{array}$ & RCT $^{*}$ & 36 & $\begin{array}{l}77.61 \\
(8.43)\end{array}$ & $\begin{array}{l}\text { A residential } \\
\text { care facility }\end{array}$ & $\begin{array}{l}\text { EG: Familiar music } \\
\text { CG: No music }\end{array}$ & Agitation & M-CMAI & $\begin{array}{l}\text { Agitation of the EG decreased } \\
\text { significantly compared to CG } \\
\text { and baseline }\end{array}$ \\
\hline 2008 & $\begin{array}{l}\text { Hicks- } \\
\text { Moore \& } \\
\text { Robinson }\end{array}$ & $\begin{array}{l}3 * 3 \\
\text { repeated } \\
\text { measures } \\
\text { design }\end{array}$ & 41 & $\begin{array}{c}84.5(6.0) \\
67-92\end{array}$ & $\begin{array}{l}\text { Three nursing } \\
\text { homes }\end{array}$ & $\begin{array}{l}\text { EG1: Favorite music (FM) } \\
\text { EG2: Hand massage (HM) } \\
\text { EG3: FM \& HM } \\
\text { CG: no treatment }\end{array}$ & Agitation & M-CMAI & $\begin{array}{l}\text { Agitation significantly decreased } \\
\text { in EG1, EG2, and EG3 immediately } \\
\text { following the intervention but no } \\
\text { significant difference in agitation } \\
\text { over the groups }\end{array}$ \\
\hline 2009 & $\begin{array}{l}\text { Park \& } \\
\text { Specht }\end{array}$ & $\begin{array}{l}\text { ABAB } \\
\text { A: music } \\
\text { B: no } \\
\text { music }\end{array}$ & 15 & $\begin{array}{c}83.40 \\
(10.26) \\
60-98\end{array}$ & Home & $\begin{array}{l}\text { Individualized } \\
\text { and preferred } \\
\text { music }\end{array}$ & Agitation & M-CMAI & $\begin{array}{l}\text { Agitation significantly decreased } \\
\text { while listening to music compared } \\
\text { to baseline. No difference in } \\
\text { agitation between music and no } \\
\text { music weeks }\end{array}$ \\
\hline
\end{tabular}




\section{Continued}

\begin{tabular}{|c|c|c|c|c|c|c|c|c|c|}
\hline 2010 & $\begin{array}{l}\text { Cooke } \\
\text { et al. }\end{array}$ & $\begin{array}{l}\text { A randomized } \\
\text { crossover design }\end{array}$ & $\begin{array}{l}47 \\
(14 / 33)\end{array}$ & $\begin{array}{l}74-94 \\
(87.2 \%)\end{array}$ & $\begin{array}{l}2 \text { LTC } \\
\text { facilities }\end{array}$ & $\begin{array}{l}\text { EG: Music group } \\
\text { CG: reading group }\end{array}$ & $\begin{array}{l}\text { Agitation } \\
\text { Anxiety }\end{array}$ & $\begin{array}{l}\text { CMAI-SF } \\
\text { RAID }^{*}\end{array}$ & $\begin{array}{l}\text { Significant increase in the } \\
\text { frequency of verbal aggression } \\
\text { over time, but no overall effect } \\
\text { of the music on agitation and } \\
\text { anxiety }\end{array}$ \\
\hline 2011 & Ho et al. & $\begin{array}{l}\text { A single } \\
\text { group pre and } \\
\text { post design }\end{array}$ & & & $\begin{array}{l}\text { A } \\
\text { hospital-based } \\
\text { nursing home }\end{array}$ & $\begin{array}{l}\text { Researcher- } \\
\text { composed music }\end{array}$ & Agitation & CMAI & $\begin{array}{l}\text { Significant decline in } \\
\text { mean agitation scores }\end{array}$ \\
\hline 2011 & Lin et al. & $\begin{array}{l}\text { A pre and } \\
\text { posttest control } \\
\text { group design }\end{array}$ & 100 & $\begin{array}{l}65-97 \\
82(6.80)\end{array}$ & $\begin{array}{l}3 \text { nursing home } \\
\text { facilities Taiwan }\end{array}$ & $\begin{array}{l}\text { EG: music } \\
\text { intervention } \\
\text { CG: normal } \\
\text { daily activities }\end{array}$ & Agitation & $\begin{array}{l}\text { Chinese } \\
\text { version of } \\
\text { CMAI }\end{array}$ & $\begin{array}{l}\text { Agitation reduced } \\
\text { significantly in the } \\
\text { experimental group }\end{array}$ \\
\hline 2011 & $\begin{array}{l}\text { Sung } \\
\text { et al. }\end{array}$ & $\mathrm{QE}$ & 55 & $\begin{array}{l}81.37 \\
(9.14)\end{array}$ & $\begin{array}{l}\text { A residential } \\
\text { care facility } \\
\text { Taiwan, } \\
\text { Australia }\end{array}$ & $\begin{array}{l}\text { EG: music } \\
\text { intervention } \\
\text { CG: usual care }\end{array}$ & $\begin{array}{l}\text { Agitation } \\
\text { Anxiety }\end{array}$ & $\begin{array}{l}\text { CMAI } \\
\text { RAID }\end{array}$ & $\begin{array}{l}\text { The reduction of agitation } \\
\text { between two groups was } \\
\text { not significantly different }\end{array}$ \\
\hline 2013 & $\begin{array}{l}\text { Dunn \& } \\
\text { Riley-Doucet }\end{array}$ & $\begin{array}{l}\text { A within-subjects, } \\
\text { repeated-measures } \\
\text { design }\end{array}$ & $5(2 / 3)$ & $\begin{array}{l}77-88 \\
(83)\end{array}$ & $\begin{array}{l}\text { An adult day } \\
\text { care center }\end{array}$ & $\begin{array}{l}\text { Non-religious } \\
\text { music group } \\
\text { and religious } \\
\text { music group }\end{array}$ & $\begin{array}{l}\text { Neuropsychiatric } \\
\text { symptoms } \\
\text { (agitation etc) }\end{array}$ & $\begin{array}{l}\text { The Agitated } \\
\text { Behavior } \\
\text { Scale }\end{array}$ & $\begin{array}{l}\text { There was no significant } \\
\text { differences between the } \\
\text { non-religious music and } \\
\text { religious music on NPS }\end{array}$ \\
\hline 2013 & Park & $\begin{array}{l}\text { One group pre and } \\
\text { posttest design }\end{array}$ & 26 & 82.19 & Own home & $\begin{array}{l}\text { Individualized } \\
\text { preferred music }\end{array}$ & Agitation & M-CMAI & $\begin{array}{l}\text { Agitation decreased while } \\
\text { listening to the music } \\
\text { compared to the baseline }\end{array}$ \\
\hline
\end{tabular}

*ADROL = Alzheimer's Disease-Related Quality of Life; CG = Control group; CMAI = Cohen-Mansfield Agitation Inventory; CMAI-SF = Cohen-Mansfield Agitation Inventory-Short Form; EG = Experimental group; M-CMAI = Modified Cohen-Mansfield Agitation Inventory; RAID = Rating Anxiety in Dementia Scale; RCT = Randomized Controlled Trial; SS = Sample Size; QE = Quasi-Experimental Design; VAS = Visual Analog Scale.

Table 3. Music therapists: the contents of music interventions $(N=7)$.

\begin{tabular}{|c|c|c|c|c|c|c|c|c|c|}
\hline Year & Authors & $\mathrm{CF}^{*} / \mathrm{TF}^{*}$ & $\begin{array}{l}\text { Music providers/ } \\
\text { agitation raters }\end{array}$ & Using tool & Timing & Duration & Frequency & $\begin{array}{l}\text { Total- } \\
\text { week }\end{array}$ & $\begin{array}{c}\text { Total- } \\
\text { sessions }\end{array}$ \\
\hline 1994 & $\begin{array}{c}\text { Clair } \\
\text { \& Bernstein }\end{array}$ & - & $\begin{array}{l}\mathrm{PI}^{*}\left(\mathrm{MT}^{*}\right) / \text { Pairs } \\
\text { of observer }\end{array}$ & Stereo system & $\begin{array}{l}\text { 10:30 am - } 11 \text { am } \\
\text { Noon (meal time) } \\
\text { 3:00 pm - 3:30 pm }\end{array}$ & $\begin{array}{c}90 \mathrm{~min} \text { (30 min } \\
\text { for each condition) }\end{array}$ & 5 days/week & $8 \mathrm{wks}$ & - \\
\hline 1996 & $\begin{array}{c}\text { Brotons \& } \\
\text { Pickett-Cooper }\end{array}$ & - & $\begin{array}{l}\text { Two MTs /MT \& } \\
\text { Caregivers (nurses, } \\
\text { nurses aids, activity } \\
\text { directors) }\end{array}$ & $\begin{array}{l}\text { A variety } \\
\text { music activities }\end{array}$ & Afternoon & $30 \mathrm{~min}$ & 2/week & - & 5 \\
\hline 2002 & $\begin{array}{l}\text { Jennings } \\
\text { \& Vance }\end{array}$ & - & $\begin{array}{l}\text { MTs/ } \\
\text { nursing assistants }\end{array}$ & - & - & $30 \mathrm{~min}$ & $1 /$ week & 4 wks & - \\
\hline 2003 & $\begin{array}{l}\text { Brotons } \\
\& \text { Marti }\end{array}$ & - & $\begin{array}{l}\text { MTs/ } \\
\text { Neuropsychologist }\end{array}$ & Group activities & $\begin{array}{l}\text { Morning ( } 7 \text { sessions): } \\
\text { patients and caregivers } \\
\text { Afternoon ( } 4 \text { sessions): } \\
\text { caregivers }\end{array}$ & - & - & - & \\
\hline 2007 & $\begin{array}{l}\text { Ledger } \\
\text { \& Baker }\end{array}$ & - & MTs/MTs & Group activity & $\begin{array}{l}3 \text { Groups in the } \\
\text { morning \& } 2 \text { in } \\
\text { the mid afternoon }\end{array}$ & $30-45 \min$ & 1 - 3/week & $\begin{array}{l}\text { At least } \\
42 \text { wks }\end{array}$ & - \\
\hline 2013 & Ridder et al. & - & MTs/MTs & & - & - & 2/week & 6 wks & 12 \\
\hline 2013 & Vink et al. & - & $\begin{array}{l}\text { Music therapy-MTs, } \\
\text { recreational } \\
\text { activities-occupational } \\
\text { therapist/nurses }\end{array}$ & Group activity & - & $40 \mathrm{~min}$ & 2/week & 2 months & 34 \\
\hline
\end{tabular}

*CF = Conceptual Framework; MT = Music Therapist; PI = Principle Investigator; TF = Theoretical Framework.

the sample size varied from 1 - 100, with 39\% of the studies having a sample size exceeding 15 (Table 2). In music therapists' studies, all studies were conducted in long-term care facilities including nursing homes, an Alzheimer's adult day care, and a Veterans Medical Center. In the non-music therapists' studies, all studies were conducted in long-term care facilities such as nursing homes, a health center, and a residential care facility, except one, and that one study was at the patient's own home.

\subsubsection{Music Interventions}

In the music therapists' studies, all studies except one provided using group activities for music interventions to 
Table 4. Non-music therapists: the contents of music interventions $(N=23)$.

\begin{tabular}{|c|c|c|c|c|c|c|c|c|c|}
\hline Year & Authors & ${ }^{*} \mathrm{CF} /{ }^{*} \mathrm{TF}$ & $\begin{array}{l}\text { Music Providers/ } \\
\text { Agitation Raters }\end{array}$ & Using tool & Timing & Duration & Frequency & Total-Week & $\begin{array}{l}\text { Total } \\
\text { Session }\end{array}$ \\
\hline 1993 & $\begin{array}{l}\text { Gerdner \& } \\
\text { Swanson }\end{array}$ & *PLST & *PI (Nurse)/PI & $\begin{array}{l}\text { An audio } \\
\text { cassette player }\end{array}$ & $\begin{array}{l}\text { 3:30 pm - 4:00 pm } \\
\text { (Prior to peak } \\
\text { level of agitation) }\end{array}$ & $30 \mathrm{~min}$ & 5 days & $\begin{array}{l}2 \text { wks (*W1: baseline, } \\
\text { W2: Music) }\end{array}$ & - \\
\hline 1994 & $\begin{array}{l}\text { Goddaer \& } \\
\text { Abraham }\end{array}$ & PLST & $\begin{array}{l}\text { PI (Nurse)/A trained } \\
\text { independent rater }\end{array}$ & A tape player & Meal time & - & - & $\begin{array}{l}4 \text { wks (W1, 3: no music } \\
\text { W2, 4: music) }\end{array}$ & - \\
\hline 1995 & $\begin{array}{l}\text { Tabloski } \\
\text { et al. }\end{array}$ & PLST & $\begin{array}{l}\text { Researchers/ } \\
\text { Researchers }\end{array}$ & A CD player & When agitation occurs & $15 \mathrm{~min}$ & $1 /$ week & 2 wks & 2 \\
\hline 1996 & $\begin{array}{l}\text { Ragneskog } \\
\text { et al. }\end{array}$ & - & Staff/authors & A tape recorder & Meal time (Dinner) & $30-45 \min$ & - & $\begin{array}{l}11 \text { wks (W1: baseline } \\
\text { W2, 3, 5 - 6, } 8 \text { - 9: music; } \\
\text { W4, 7, } 10 \text { - 11: no music) }\end{array}$ & 40 \\
\hline 1997 & Denney & PLST & - & A tape player & $\begin{array}{l}\text { Meal time: } \\
\text { 11:45 am - 1:15 pm }\end{array}$ & $90 \mathrm{~min}$ & Daily & $\begin{array}{l}4 \text { wks (W1, 3: No music } \\
\text { W2, 4: Music) }\end{array}$ & 14 \\
\hline 1997 & Gerdner & *IMIA & - & & Peak agitation time & $30 \mathrm{~min}$ & 2/week & $\begin{array}{l}6 \text { wks (classical music), } \\
2 \text { wks (no music), } \\
6 \text { wks (religious music) }\end{array}$ & \\
\hline 1997 & $\begin{array}{l}\text { Thomas } \\
\text { et al. }\end{array}$ & - & *CNA/CNA & A tape recorder & Bathing time & - & - & - & 3 \\
\hline 1998 & $\begin{array}{l}\text { Clark } \\
\text { et al. }\end{array}$ & - & $*$ RAs/RAs & $\begin{array}{l}\text { A CD radio } \\
\text { cassette recorder }\end{array}$ & Bathing time & - & - & $\begin{array}{l}2 \text { wks: no music } \\
2 \text { wks: music }\end{array}$ & 10 \\
\hline 2000 & Gerdner & IMIA & RAs/RAs & $\begin{array}{l}\text { A portable audio } \\
\text { cassette player }\end{array}$ & Peak agitation time & $30 \mathrm{~min}$ & 2/week & $\begin{array}{l}\text { W2 - 4: baseline } \\
\text { W5 - 10: Individualized music, } \\
\text { W11 - 12: wash-out, } \\
\text { W13 - 18: Classical music }\end{array}$ & \\
\hline 2001 & $\begin{array}{l}\text { Ragneskog } \\
\text { et al. }\end{array}$ & - & Staff/Researchers & $\begin{array}{l}\text { A prerecorded } \\
\text { cassette recorder }\end{array}$ & Most agitated time & $30 \mathrm{~min}$ & & $\begin{array}{l}\text { Control period: no music, } \\
2 \text { periods: individualized music, } \\
1 \text { period: classical music }\end{array}$ & $4-5$ \\
\hline 2004 & $\begin{array}{l}\text { Richeson } \\
\text { \& Neill }\end{array}$ & $* \mathrm{NDB}$ & $\begin{array}{l}\text { Therapeutic recreation } \\
\text { specialist, nursing } \\
\text { staff, \& researchers/ } \\
\text { researchers }\end{array}$ & A CD player & $\begin{array}{l}\text { Meal time: } \\
5 \text { pm - } 6 \text { pm. }\end{array}$ & $60 \mathrm{~min}$ & 4/week & $\begin{array}{l}\text { W1: baseline } \\
\text { W2: Music }\end{array}$ & - \\
\hline 2005 & Gerdner & $\begin{array}{l}\text { PLST, } \\
\text { IMIA }\end{array}$ & CNA/CNA & $\begin{array}{l}\text { A portable } \\
\text { CD player }\end{array}$ & Peak agitation time & $30 \mathrm{~min}$ & Daily & $\begin{array}{l}\text { Month 1: baseline, } \\
\text { month } 2 \text { - 3: music }\end{array}$ & - \\
\hline 2005 & $\begin{array}{l}\text { Hicks- } \\
\text { Moore }\end{array}$ & - & -/researchers & - & Meal time & - & Daily & $\begin{array}{l}\text { W1, 3: no music, } \\
\text { W2, 4: music }\end{array}$ & - \\
\hline 2006 & $\begin{array}{l}\text { Sung } \\
\text { et al. }\end{array}$ & - & Trained nurses/- & - & Mid-afternoon & $30 \mathrm{~min}$ & 2/week & 6 wks & - \\
\hline 2006 & $\begin{array}{l}\text { Sung } \\
\text { et al. }\end{array}$ & - & $\begin{array}{l}\text { A nursing researcher } \\
\text { \& RAs/nursing staff }\end{array}$ & A CD player & $3: 00$ - 3:30 pm & $30 \mathrm{~min}$ & 2/week & 4 wks & 8 \\
\hline 2008 & $\begin{array}{l}\text { Hicks- } \\
\text { Moore \& } \\
\text { Robinson }\end{array}$ & - & RNs or Faculty/RA & $\begin{array}{l}\text { A portable } \\
\text { CD player }\end{array}$ & $\begin{array}{l}\text { when agitation } \\
\text { occurred }\end{array}$ & $10 \mathrm{~min}$ & & - & 1 \\
\hline 2009 & $\begin{array}{l}\text { Park \& } \\
\text { Specht }\end{array}$ & PLST & $\begin{array}{l}\text { Family } \\
\text { caregivers/family } \\
\text { caregivers }\end{array}$ & $\begin{array}{l}\text { A portable } \\
\text { CD player }\end{array}$ & Peak agitation time & $30 \mathrm{~min}$ & 2/week & $\begin{array}{l}\text { W1 - 2, } 5 \text { - 6: music } \\
\text { W3 - 4, } 7 \text { - 8: no music }\end{array}$ & 8 \\
\hline 2010 & $\begin{array}{l}\text { Cooke } \\
\text { et al. }\end{array}$ & - & Musicians/RAs & $\begin{array}{l}\text { Guitar, } \\
\text { pre-recorded } \\
\text { instrumental } \\
\text { music }\end{array}$ & Morning & $40 \mathrm{~min}$ & 3/week & $\begin{array}{l}\text { W1 - 8: music, } \\
\text { W9 - 13: wash out, } \\
\text { W14 - 21: reading }\end{array}$ & 16 \\
\hline 2011 & Ho et al. & - & Researchers & A CD player & Meal time & $60 \mathrm{~min}$ & daily & 4 wks & 28 \\
\hline 2011 & $\begin{array}{l}\text { Lin } \\
\text { et al. }\end{array}$ & - & Researcher & $\begin{array}{l}\text { Group } \\
\text { activities }\end{array}$ & - & $30 \mathrm{~min}$ & 2/week & 6 wks & 12 \\
\hline 2011 & $\begin{array}{l}\text { Sung } \\
\text { et al. }\end{array}$ & - & RAs & $\begin{array}{l}\text { Percussion } \\
\text { instruments }\end{array}$ & Mid afternoon & $30 \mathrm{~min}$ & 2/week & 6 wks & 12 \\
\hline 2013 & $\begin{array}{l}\text { Dunn \& } \\
\text { Riley- } \\
\text { Doucet }\end{array}$ & - & $\begin{array}{l}\text { Formal } \\
\text { caregivers }\end{array}$ & A CD player & $\begin{array}{l}\text { Any time in a } \\
\text { private corner }\end{array}$ & - & M & $\begin{array}{l}\text { W1 - 2: non-religious music } \\
\text { W3 - 4: religious music } \\
\text { (or the other way) }\end{array}$ & 34 \\
\hline 2013 & Park & - & PI & A CD player & $\begin{array}{l}\text { Peak } \\
\text { agitation time }\end{array}$ & $30 \mathrm{~min}$ & 2/week & 2 wks & 4 \\
\hline
\end{tabular}

*CAN = Certified Nurse Assistants; IMIA = Individualized Music Intervention on Agitation; NDB = Need-Driven Dementia-Compromised Behavior; PI = Principle Investigator; PLST = Progressively Lowered Stress Threshold Model; RAs = Research Assistants; W = Week. 
control agitation and one study provided individualized music therapy (Table 1). The music interventions included diverse music activities such as singing songs, music listening, playing rudimentary instruments, dance/movement, composition/improvisation, music games, and music relaxation exercises. These diverse music activities allowed clients to enjoy the music depending on their functional status. In the non-music therapists' studies, $39 \%$ of the studies provided individualized music to meet the patients' music preferences and the patients' music favorites were assessed before offering music intervention (Table 3). The music interventions included playing the recorded music, such as relaxing/quiet music, classical music, rock music or religious music as background music. The genre of recorded music was determined based on the researchers' own plans or the clients’ music preferences.

\subsubsection{Instruments}

In the music therapists' studies, the agitation level was measured using diverse measurements, including, most commonly, the Cohen-Mansfield Agitation Inventory, the Disruptive Behavior Rating Scales, the Neuropsychiatry Inventory, video analysis, and interviews. In the non-music therapists' studies, the agitation level was also measured using diverse instruments: the Cohen-Mansfield Agitation Inventory (CMAI) was the most commonly used assessment, followed by an agitation behavior scale, video analysis, and observation. In both groups, the CMAI was most commonly used after being modified in several studies [16] [18] [20].

\subsubsection{Outcomes}

In the music therapists' studies, 4 out of 7 studies showed significant decreases in agitation during the presence of music compared with the baseline and the other group, and the other 3 studies reported no significant difference between the groups. The authors assumed that the absence of an effect from background music on agitation was because the music was selected without guidance as to the clients' musical tastes. Also, the lack of significant difference in agitation between the groups over time may indicate that music intervention has only a short-term effect on agitation. In the non-music therapists' studies, most studies showed a decrease in agitation with music compared with the baseline but only 5 studies showed a significantly reduced agitation with a music group, compared with the other group (preferred music other than classical music or no music).

\subsection{The Contents of Music Intervention}

In the music therapists' studies, no study explained a conceptual framework or theoretical framework, but in the non-music therapists' studies, 9 studies presented a theoretical framework, such as the progressively lowered stress threshold model, a mid-range theory of individualized music intervention for agitation, and the need-driven dementia-compromised behavior model. In the non-music therapists' studies, music interventions were provided by nurses, certified nursing assistants, musicians, therapeutic recreation specialists, research assistants, staff, and family caregivers.

In the music therapists' studies, the timing of providing music interventions were morning, noon (meal time), or afternoon. In the non-music therapists' studies, the timing of providing music interventions were diverse, such as peak agitation time, meal time, bathing time, morning, afternoon, and any time. In the music therapists' studies, the duration of music intervention was 30 to 45 minutes for a session. In the non-music therapists' studies, the duration of music intervention was more diverse from 15 to 90 minutes, but the most common duration of music interventions was 30 minutes (14 studies in both groups). The rationale of offering music intervention for 30 minutes was in the recognition of the limited attention span in patients with dementia. Finally, the frequency of offering the music intervention was diverse in the music therapists' and non-music therapists' studies and they offered the intervention most commonly two times a week (11 studies).

\section{Discussion}

The present study was undertaken to review the differences of music interventions to control agitation in patients with dementia between music therapists and non-music therapists, with the aim of providing practical ideas to improve the interventions for each group. Music-intervention studies by music therapists were inclined to have more subjects compared with non-music therapists. A more reasonable sample size needs to be included using calculation of the sample size, and furthermore the rationale for determining the sample size needs to be presented in the studies. All studies except two studies took place in nursing homes, health center, nursing facil- 
ity, long-term care facility, skilled care facility, and so on and only two studies were performed in the patients' own homes [17]. Thus, more music intervention studies need to be conducted on different communities including patients' own home for the future studies.

In terms of the findings about music interventions, 4 out of the 7 music therapists' studies reported significant effects of music interventions compared with the other group. In non-music therapists' studies, most studies showed significant decreases in agitation compared with the baseline, but only 5 out of 27 studies showed significant effects of music interventions to the comparison groups. For future studies, a comparison about the effects of music on agitation with different types of music needs to be conducted using a more sophisticated level of research design, such as randomized controlled trials.

The Cohen-Mansfield Agitation Inventory (CMAI) is not only the most commonly used instrument to measure agitation in the music therapists' studies but also for the non-music therapists. To increase the validity of the study findings, another measurement, such as video analysis, observation or interviews with caregivers, might be added to measure agitation for both types of music providers. In addition, while music therapists provided music interventions any time, such as morning, noon, or afternoon, the non-music therapists performed music interventions mostly at peak agitation time, mealtime, or bathing time [17] [24] [25]. The reasons for performing the intervention at those times are assumed to be that meal time and bathing time are important moments to care for patients with dementia for the usual care and is also the time when it is likely for agitation to often occur, so that the care providers want to control agitation during the meal time and bathing time with music.

For the contents of music interventions, the music therapists' studies provided music interventions including diverse methods with group activities, such as singing, playing instruments, improvising, or dancing. On the other hand, the non-music therapists' studies usually provided music interventions using recorded music for background music and they were likely to perform the intervention for individual patients with dementia. The differences of offering music intervention methods between the studies are based on their educational backgrounds and clinical experiences. For future studies, to consider the pros and cons of each music provider's education background and clinical experiences, multi-disciplinary music intervention studies need to be carried with music therapists, nurses, or recreational therapists.

\section{Conclusion}

The current study reviewed the differences in the contents of music intervention studies to control agitation between the music therapists and non-music therapists, with the goal of providing practical guidelines for music providers. The music therapists' studies had a more reasonable sample size, various music activities, and the comparison groups. Whereas, the non-music therapists' studies had a more theoretical framework of music interventions, used the recorded music for the background music, provided music at more diverse times such as peak agitation time, meal time, or bathing time. For future studies, multi-disciplinary music intervention studies including music therapists, nurses, recreational therapists, occupational therapists and so on are recommended to improve the effects of music intervention on agitation

\section{Funding}

This research was supported by Basic Science Research Program through the National Research Foundation of Korea (NRF) funded by the South Korean Ministry of Education (2015R1D1A3A01017843).

\section{References}

[1] Imperio, K. and Pusey-Reid, E. (2006) Cognitive and Neurological Function. In: Meiner, S. and Lueckenotte, A., Eds., Gerontological Nursing, Mosby, St. Louis, 653-692.

[2] Rodney, V. (2000) Nurse Stress Associated with Aggression in People with Dementia: Its Relationship to Hardiness, Cognitive Appraisal and Coping. Journal of Advanced Nursing, 31, 172-180.

[3] Sourial, R., McCusker, J., Cole, M. and Abrahamowicz, M. (2001) Agitation in Demented Patients in an Acute Care Hospital: Prevalence, Disruptiveness, and Staff Burden. International Psychogeriatrics, 13, 183-197. http://dx.doi.org/10.1017/S1041610201007578

[4] Yaffe, K., Fox, P., Newcomer, R., Sands, L., Lindquist, K., Dane, K. and Covinsky, K.E. (2002) Patient and Caregiver Characteristics and Nursing Home Placement in Patients with Dementia. JAMA: Journal of the American Medical Association, 287, 2090-2097. http://dx.doi.org/10.1001/jama.287.16.2090 
[5] Buchalter, E. and Lantz, M. (2001) Treatment of Impulsivity and Aggression in a Patient with Vascular Dementia. Geriatrics, 56, 53-54.

[6] Kyomen, H.H. and Gottlieb, G.L. (2005) The Cost of Psychotropic Drug Use for Elderly. In: Salzman, C., Ed., Clinical Geriatric Psychopharmacology, 4th Edition, Lippincott Williams \& Wilkins, Philadelphia, 49-60.

[7] Association, A.M.T., in Membership Brochure, 1997.

[8] Brotons, M. and Koger, S.M. (2000) The Impact of Music Therapy on Language Functioning in Dementia. Journal of Music Therapy, 37, 183-195. http://dx.doi.org/10.1093/jmt/37.3.183

[9] Brotons, M. and Marti, P. (2003) Music Therapy with Alzheimer's Patients and Their Family Caregivers: A Pilot Project. Journal of Music Therapy, 40, 138-150. http://dx.doi.org/10.1093/jmt/40.2.138

[10] Clair, A.A. (2002) The Effects of Music Therapy on Engagement in Family Caregiver and Care Receiver Couples with Dementia. American Journal of Alzheimer's Disease \& Other Dementias, 17, 286-290. http://dx.doi.org/10.1177/153331750201700505

[11] Ragneskog, H., Kihlgren, M., Karlsson, I. and Norberg, A. (1996) Dinner Music for Demented Patients: Analysis of Video-Recorded Observations. Clinical Nursing Research, 5, 262-282. http://dx.doi.org/10.1177/105477389600500302

[12] Sherratt, K., Thornton, A. and Hatton, C. (2004) Emotional and Behavioural Responses to Music in People with Dementia: An Observational Study. Aging \& Mental Health, 8, 233-241. http://dx.doi.org/10.1080/13607860410001669769

[13] Suzuki, M., Kanamori, M., Watanabe, M., Nagasawa, S., Kojima, E., Ooshiro, H. and Nakahara, D. (2004) Behavioral and Endocrinological Evaluation of Music Therapy for Elderly Patients with Dementia. Nursing \& Health Sciences, 6, 11-18. http://dx.doi.org/10.1111/j.1442-2018.2003.00168.x

[14] Gerdner, L.A. (2000) Effects of Individualized versus Classical "Relaxation” Music on the Frequency of Agitation in Elderly Persons with Alzheimer’s Disease and Related Disorders. International Psychogeriatrics, 12, 49-65. http://dx.doi.org/10.1017/S1041610200006190

[15] Gerdner, L.A. (2005) Use of Individualized Music by Trained Staff and Family: Translating Research into Practice. Journal of Gerontological Nursing, 31, 22-30. http://dx.doi.org/10.3928/0098-9134-20050601-08

[16] Jennings, B. and Vance, D. (2002) The Short-Term Effects of Music Therapy on Different Types of Agitation in Adults with Alzheimer's. Activities, Adaptation \& Aging, 26, 27-33. http://dx.doi.org/10.1300/J016v26n04_03

[17] Park, H. and Pringle Specht, J.K. (2009) Effect of Individualized Music on Agitation in Individuals with Dementia Who Live at Home. Journal of Gerontological Nursing, 35, 47-55. http://dx.doi.org/10.3928/00989134-20090706-01

[18] Sung, H., Chang, A.M. and Abbey, J. (2006) The Effects of Preferred Music on Agitation of Older People with Dementia in Taiwan. International Journal of Geriatric Psychiatry, 21, 999-1000. http://dx.doi.org/10.1002/gps.1585

[19] Richeson, N.E. and Neill, D.J. (2004) Therapeutic Recreation Music Intervention to Decrease Mealtime Agitation and Increase Food Intake in Older Adults with Dementia. American Journal of Recreation Therapy, 3, 37-41.

[20] Thomas, D.W., Heitman, R. and Alexander, T. (1997) The Effects of Music on Bathing Cooperation for Residents with Dementia. Journal of Music Therapy, 34, 246-259. http://dx.doi.org/10.1093/jmt/34.4.246

[21] Goodall, D. and Etters, L. (2005) The Therapeutic Use of Music on Agitated Behavior in Those with Dementia. Holistic Nursing Practice, 19, 258-262. http://dx.doi.org/10.1097/00004650-200511000-00005

[22] Lou, M. (2001) The Use of Music to Decrease Agitated Behaviour of the Demented Elderly: The State of the Science. Scandinavian Journal of Caring Sciences, 15, 165-173. http://dx.doi.org/10.1046/j.1471-6712.2001.00021.x

[23] Sung, H. and Chang, A.M. (2005) Use of Preferred Music to Decrease Agitated Behaviours in Older People with Dementia: A Review of the Literature. Journal of Clinical Nursing, 14, 1133-1140. http://dx.doi.org/10.1111/j.1365-2702.2005.01218.x

[24] Lin, Y., Chu, H., Yang, C.-Y., Chen, C.-H., Chen, S.-G., Chang, H.-J., et al. (2011) Effectiveness of Group Music Intervention against Agitated Behavior in Elderly Persons with Dementia. International Journal of Geriatric Psychiatry, 26, 670-678. http://dx.doi.org/10.1002/gps.2580

[25] Hicks-Moore, S.L. (2005) Relaxing Music at Mealtime in Nursing Homes: Effect on Agitated Patients with Dementia. Journal of Gerontological Nursing, 31, 26-32. http://dx.doi.org/10.3928/0098-9134-20051201-07 